García-Sanz, M.P. (2014). La evaluación de competencias en Educación Superior mediante rúbricas: un caso práctico. Revista Electrónica Interuniversitaria de Formación del Profesorado, 17 (1), 87-106.

DOI: http://dx.doi.org/10.6018/reifop.17.1. 198861

\title{
La evaluación de competencias en Educación Superior mediante rúbricas: un caso práctico
}

\author{
Mari Paz García Sanz \\ Universidad de Murcia
}

\section{Resumen}

En educación superior, la evaluación es la herramienta que permite valorar el nivel de logro de las competencias transversales y específicas que los estudiantes tienen que adquirir en las diferentes titulaciones universitarias, actuando además de elemento regulador del aprendizaje. Se trata de realizar una evaluación no sólo del desarrollo de competencias, sino también para el desarrollo de las mismas.

Para demostrar las competencias adquiridas por los estudiantes en relación a cuánto han aprendido y lo bien que lo han aprendido, puede recurrirse a las rúbricas, mediante las cuales es posible valorar aspectos complejos, imprecisos y subjetivos, aportando una evaluación fácilmente interpretable, justa y transparente para profesores y estudiantes. A modo de ejemplo, en este artículo se presenta una rúbrica para evaluar el portafolios de una asignatura universitaria de la titulación de Grado en Pedagogía.

\section{Palabras clave}

Educación superior; competencia; evaluación de competencias; rúbrica.

\section{Assessing competencies in higher education by rubrics: a case study}




\section{Abstract}

In higher education, the assessment is the tool to assess the level of achievement of the transversal and specific competencies that students must acquire in different university degrees, besides acting regulatory element of learning. This is not only an assessment of competencies development, but also to develop them.

To demonstrate the competencies acquired by students in relation to how much they have learned and how well they have learned it, you can resort to the rubrics by which it is possible to value complex, imprecise and subjective aspects, providing an easily interpretable, fair and transparent assessment for teachers and students. As an example, this article presents a rubric to evaluate the portfolio of a university subject the Degree in Pedagogy.

\section{Key words}

Higher education; competence; competency assessment; rubric.

\section{Introducción}

El modelo educativo que defiende el Espacio Europeo de Educación Superior (EEES) está centrado en el aprendizaje de competencias por parte del estudiante, de manera que todos los aspectos curriculares y organizativos se definen en torno a las competencias que tiene que adquirir el alumnado (Holmes, 2001; Biggs, 1999 y 2004; Colás, Jiménez y Villaciervos 2005; Imbernón, 2006; Zabalza, 2006; Martín y Wolff, 2011; López Ruiz, 2011; García Sanz, 2011; Martín del Pozo et al, 2013).

Este nuevo modelo competencial (García Sanz, 2011) sustituye a una enseñanza tradicionalmente centrada en la transmisión de conocimientos y conlleva que el profesorado universitario se replantee su actuación como docente. Los profesores necesitan, pues, una sólida formación -especialmente pedagógica- que le permitan ser capaz de practicar una enseñanza de calidad en los nuevos Grados y Postgrados. Para ello, tendrán que dotarse de una serie de competencias (Zabalza, 2003; Rodríguez Espinar, 2003; Perrenoud, 2004; Galán, 2007 , etc.) que le ayuden a poder conducir al estudiante hacia el desarrollo de sus competencias académicas y profesionales con los recursos disponibles, especialmente en estos momentos de crisis económica (King, Marginson, y Naidoo, 2011; Masacre y Cantwell, 2012; Moran, 2012; Livingston, 2012; Menter y Hulme, 2012; Draper, 2012). De acuerdo con Rodríguez Izquierdo (2003), en los tiempos actuales hay que reconceptualizar la docencia, los profesores deben reaprender a enseñar y los estudiantes han de reaprender a aprender para adaptar la educación universitaria a las necesidades actuales.

La evaluación es uno de los elementos clave del currículum, por lo que, en continua interrelación con el resto, de acuerdo con el modelo competencial mencionado, debe ir encaminada a poner de manifiesto el logro de competencias previamente determinadas. Por ello, al planificar las competencias que queremos que desarrollen los estudiantes, no sólo hay que pensar en cómo enseñarlas, sino también en cómo evaluarlas. Ahora bien, dado que, como venimos señalando, las prácticas de enseñanza universitaria están cambiando, lo mismo se puede afirmar cuando nos referimos a las prácticas de evaluación (Guilles, Detroz y Blais, 2011). Se hace pues necesario conocer y saber aplicar un sistema de evaluación continuo, variado y formativo (Inda, Álvarez y Álvarez, 2008), dentro de lo que se ha venido a denominar la aparición de una nueva cultura de la evaluación (Rust, 2007). 


\section{La evaluación centrada en competencias}

De acuerdo con Villa y Poblete (2011), un sistema de evaluación de calidad garantiza que tanto estudiantes como empleadores puedan confiar en una formación adecuada cuando los primeros han obtenido buenas calificaciones. De esta forma, los empleadores pueden recibir la garantía de que los servicios prestados por los profesionales son eficaces y seguros (Mcgaghie, 1991). Además, algunos estudios han demostrado la solicitud por parte de los estudiantes de evaluar todas las tareas encomendadas por sus profesores (Lemanski, 2011). Y es que lo que no se evalúa, no se valora. Por eso, cuando les pedimos a los estudiantes que consulten una determinada información, su primera pregunta es: ¿entra para el examen?.

Como ya hemos indicado en otras ocasiones (García Sanz, 2011 y 2012), la evaluación la entendemos como un proceso sistemático, intencional y continuo de recogida de información, análisis, interpretación y valoración de la misma, en base a criterios, cuya finalidad es la toma de decisiones en relación al objeto evaluado.

Dentro de lo que hemos venido a denominar "El ciclo de intervención educativa" (ibídem), la evaluación se convierte en un proceso que activa, orienta y afecta (Huertas, 2009), ya que la evaluación que interesa es aquella que active al estudiante hacia el aprendizaje, que le oriente hacia la mejora y que le afecte positivamente en relación a los esfuerzos realizados. Para ello, hay que informar al estudiante de manera continua de sus progresos en relación con las competencias que va desarrollando durante el proceso de aprendizaje y no únicamente al final, considerando la evaluación de resultados como la consecuencia de todas las actividades realizadas en el transcurso de la asignatura.

De acuerdo con Castro (2010:118)), "la evaluación de competencias se puede definir como un procedimiento en el que se requiere que el estudiante complete tareas o procesos en los que se demuestre su habilidad para aplicar conocimiento y destrezas o aplicar conocimientos en situaciones simuladas similares a la vida real". Por lo tanto, la evaluación de competencias supone valorar la capacidad del alumno para dar respuesta a situaciones más o menos reales con las que, con muchas probabilidades, se va a encontrar en el futuro, aunque evidentemente, nunca del mismo modo en que han sido aprendidas, ya que las combinaciones de problemas y contextos reales pueden ser infinitas (Zabala y Arnau, 2007). De cualquier modo, las competencias se demuestran en la acción, por lo que únicamente pueden ser evaluables si se determinan actividades de aplicación de las mismas.

La evaluación de competencias, por sus propias características e implicaciones, constituye una de las tareas más importantes en los procesos educativos universitarios, especialmente por su propia utilidad formativa y sus consecuencias socioprofesionales, como son la certificación, reconocimiento, convalidación de experiencia, etc. (Tejada, 2011). Por ello, la evaluación debe tener una relevancia especial en la educación universitaria (Cowan, 2010), especialmente porque constituye la herramienta más influyente en el aprendizaje de los estudiantes (Gibbs, 1999; Biggs, 2004). Asimismo, el contexto en el que se manifiestan las mismas constituye un elemento clave para su adecuación, ya que competencias en diferentes contextos requieren diferentes combinaciones de conocimientos, habilidades y actitudes (Gairín et al, 2009), por lo que el ámbito profesional en el que van a ser aplicadas ha de ser tenido muy en cuenta.

Puesto que, el concepto de competencia integra conocimientos, habilidades, destrezas, actitudes, valores y normas (García Sanz, 2008), siguiendo a Medina-Rivilla, DomínguezGarrido y Sánchez-Romero (2013), la evaluación de competencias "implica estimar el dominio de los saberes más relevantes, su aplicación para resolver los problemas de la 
profesión y el avance en los valores y actitudes más coherentes con la deontología exigida para su desempeño" (p. 242). Asimismo, para Mateo (2007), en el modelo educativo que propugna la Convergencia Europea, el proceso evaluativo debe aportar información proveniente de las observaciones directas de las ejecuciones realizadas por los estudiantes y ha de estar orientado, no a la reproducción de la respuesta, sino a la construcción de la misma mediante razones que expliquen el por qué de dicha respuesta. Para ello, las actividades evaluativas deben reflejar aquellos contenidos sujetos a evaluación que son verdaderamente importantes para el aprendizaje del alumnado, es decir, los que ponen de manifiesto la adquisición de competencias, a la vez que deben constituir una herramienta de mejora del propio aprendizaje y de apoyo al proceso de enseñanza. Se trata de pasar de una "evaluación del aprendizaje" a una "evaluación para el aprendizaje" (López Pastor, 2009), e incluso a una evaluación "como aprendizaje" (Torrance, 2007), ya que una evaluación de calidad puede enseñar más que muchas otras actividades docentes.

Ibarra, Rodríguez y Gómez (2012: 210), definen la evaluación orientada al aprendizaje "como un constructo teórico, basado en la práctica universitaria, que hace hincapié en considerar la evaluación como un proceso interrelacionado con el aprendizaje, a partir del cual, no solo se puede extraer información útil para el estudiante, sino que se posibilita la promoción del desarrollo de competencias valiosas para su presente académico y desarrollo profesional". Desde esta perspectiva, las actividades de evaluación contribuyen positivamente al desarrollo del conocimiento y a una mayor disposición hacia el aprendizaje mas allá de las aulas universitarias (Boud y Falchikov, 2006). El objetivo es evaluar para ayudar al estudiante a mejorar el dominio de las competencias, por lo que es necesario conocer cuáles son sus dificultades para establecer estrategias de aprendizaje apropiadas para superarlas. De hecho, cuando los estudiantes perciben que los profesores se preocupan por la docencia y por establecer procedimientos de evaluación informativos, completos, justos y transparentes que les ayuden en sus aprendizajes, suelen mostrarse receptivos hacia la materia y motivados en la realización de las tareas.

Para Álvarez (2008), la evaluación orientada al aprendizaje debe apoyarse en tres aspectos fundamentales:

- Plantear las actividades de evaluación como tareas de aprendizaje.

- Implicar a los estudiantes en la evaluación.

- Ofrecer los resultados de la evaluación a modo de fedback.

En relación a los instrumentos de recogida de información para evaluar el nivel de logro de las competencias desarrolladas por lo estudiante, es crucial tener muy claro en qué consiste aquello que se pretende evaluar: la competencia, ya que, como se ha expresado más arriba, profesores e investigadores aun no se han puesto muy de acuerdo en su significado, lo cual está originando un gran desconcierto y preocupación (Gijbels, 2011). Por lo tanto, de acuerdo con Baartman y Braun (2011), para evaluar el desarrollo de competencias es de suma importancia especificar claramente lo que se entiende por este concepto.

De cualquier manera, para la evaluación del aprendizaje de los estudiantes es necesario utilizar una gran variedad de instrumentos de recogida de información acordes con las competencias consideradas (Baartman, Bastiaens, Kirschner y Van der Vleuten, 2007; Gerard y BIEF, 2008; Martínez Clares y Echeverría, 2009; Harman y McDowell, 2011; Frost, de Pont y Brailsford, 2012) para atender al principio de multivariedad y triangulación instrumental, ya que, como hemos mencionado, la misma no puede reducirse a comprobar una simple reproducción de conocimientos, sino que ha de ser capaz de valorar el desarrollo de las competencias explicitadas $y$, por lo tanto, poner de manifiesto qué saben los alumnos, qué 
saben hacer y cómo saben ser y estar. Ello contribuirá a que los estudiantes, además de poner de manifiesto lo que han aprendido, lo demuestren de modo eficiente, aun siendo conscientes de que lo que el alumnado sabe y sabe hacer no suele coincidir fielmente con lo que demuestra en una situación de evaluación. En cualquier caso, la evaluación de una competencia ha de realizarse mediante instrumentos que pongan de manifiesto el grado de logro de la misma, en función del dominio de contenidos propios del constructo evaluado. Por lo tanto, la evaluación de competencias requiere el empleo de distintos instrumentos de recogida de información sobre el progreso del alumnado complementarios, de manera que dicha información sea completa y permita la emisión de juicios de valor más acertados, precisos y justos (López Ruiz, 2011). Asimismo, es aconsejable la utilización de procedimientos de autoevaluación, evaluación recíproca entre estudiantes y coevaluación entre profesorado y alumnado, de manera que se le otorgue una mayor participación a todos los implicados en el proceso evaluativo (Black y William, 1998; Dochy, Segers y Sluijmans, 1999; Echeberría, 2002; Taras, 2002 y 2010; McDonald y Boud, 2003; Falchikov, 2005; Bretones, 2008; Rodríguez, Ibarra y Gómez, 2011; Hernández, 2012; Trevitt, Breman y Stocks, 2012; Medina-Rivilla, Domínguez-Garrido y Sánchez-Romero, 2013, entre otros).

Del mismo modo, hay que explicitar al alumnado la ponderación de cada uno de los instrumentos de evaluación, es decir, no es suficiente con señalar que a los estudiantes se les va a evaluar con un examen y que un determinado trabajo, incluso la asistencia y participación en clase, puede subir o bajar la calificación. Recordemos que los discentes únicamente trabajan y valoran aquello que saben que les va a repercutir de una forma explícita en su evaluación final. Igualmente, el profesorado debe especificar claramente cuáles son los criterios de calidad (Baartman, Bastiaens, Kirschner y Van der Vleuten, 2007; Payne y Brown, 2011; Van der Schaaf, Baartman y Prins, 2012) que se van a tomar como punto de referencia dentro de cada instrumento de evaluación para valorar el desarrollo de las competencias en los estudiantes en forma de indicadores de logro. Es conveniente que dichos criterios de calidad sean consensuados entre profesores y alumnos, deben estar claramente formulados de manera que sean muy comprensibles para los estudiantes (Rust, Price y O'Donovan, 2003) y recoger de forma válida y fiable aquellos aspectos que, de forma relevante, son capaces de poner de manifiesto la cantidad y calidad de los aprendizajes que se han adquirido en relación con las competencias a desarrollar. Los criterios de calidad guían al profesor en el proceso de evaluación del alumnado, pero el papel más importante lo adquieren en la medida que los estudiantes saben en base a qué van a ser evaluados, lo cual también ayuda en el proceso de autoevaluación y evaluación recíproca a los que nos hemos referido anteriormente.

Por otra parte, cada instrumento de recogida de información está asociado a una o varias competencias, es decir, la elección de dichos instrumentos no es arbitraria, sino que se encuentra en función del tipo de competencia o competencias a evaluar. Por lo tanto, los criterios de calidad ponen de manifiesto el nivel de logro de las competencias relacionadas con cada actividad de evaluación. En este sentido, puede ocurrir que los criterios demuestren la calidad de un trabajo, examen, exposición, etc., pero no ser capaces de discriminar sobre la adquisición o no de cada una de las competencias asociadas a dichas técnicas de recogida de información. En este caso, otra posibilidad más directa es partir de la competencia a evaluar y determinar los criterios que demuestran el logro de la misma.

\section{Las rúbricas como herramienta para evaluar competencias}

Recordemos que la adquisición de una determinada competencia no es cuestión de todo o nada, sino que su desarrollo conlleva distintos niveles de logro o desempeño. Para evaluar y 
sistematizar el grado de dominio de las competencias -bien de forma aislada o integrada en un determinado procedimiento de evaluación-, puede recurrirse a las rúbricas, mediante las cuales es posible valorar aspectos complejos, imprecisos y subjetivos, aportando una evaluación fácilmente interpretable, justa y transparente para profesores y estudiantes. Según Gairín et al (2009) la rúbrica ayuda a los profesores a definir la excelencia y el plan de instrucción, alinea los objetivos del currículo y la propuesta de evaluación, ayuda a ser cuidadosos, honestos y consistentes en las calificaciones, reduce el tiempo utilizado para evaluar el trabajo del estudiante, puede promover la consistencia entre las expectativas y los resultados de cursos, departamentos, etc. En los estudiantes, la rúbrica aclara las expectativas de los profesores, crea un alto nivel de exigencia con una realización de calidad claramente indicada, ayuda en la autoevaluación y la evaluación recíproca y proporciona un feedback orientado que identifica cómo y dónde hay que mejorar. De acuerdo con Brough y Pool (2005) las rúbricas permiten a los estudiantes juzgar la calidad de su trabajo y facilitar las formas en que se podría mejorar.

Los orígenes de la rúbrica se sitúan en 1912, en un estudio realizado por Ernest C. Noyes, quien, ante la necesidad de valorar los textos escritos de manera que tuviesen el mismo significado para todas las personas y en todos los lugares y no depender de la opinión subjetiva de cada una, elaboró una especie de rúbrica denominada: "Scale for the Measurement of Quality in English composition by Young People", más comúnmente conocida como la "Hillegas Scale". Estas escalas fueron creadas fuera de las escuelas, generalmente en universidades, para ser posteriormente utilizadas en dichos centros educativos y permitir comparaciones entre estudiantes, profesores, clases, escuelas, ciudades, y así sucesivamente.

Las rúbricas constituyen un conjunto de criterios de calidad relacionados con la competencia o competencias a evaluar, determinados por descriptores o indicadores que suponen distintos niveles de logro o desempeño de los mismos. Dichos niveles han de poner de manifiesto no sólo el incremento cuantitativo de los estudiantes, sino también el salto cualitativo, es decir, demostrar cuánto han aprendido y lo bien que han aprendido.

En los últimos años son muchas las experiencias internacionales que han demostrado los beneficios que conlleva el empleo de las rúbricas (Wilson y Onwuegbuzie, 1999; Hafner y Hafner, 2003; Andrade y Du, 2005; Campbell, 2005; Dunbar, Brooks y Kubicka-Miller, 2006; Jonsson y Svingby, 2007; Mansilla, Duraisingh, Wolfe y Haynes, 2009; Vandenberg, Stollak, McKeag y Obermann, 2010; Tractenberg, Umans y McCarter, 2010; Timmerman, Strickland, Johnson y Payne, 2011; Rochele, Tractenberg, Kevin y FitzGerald, 2012, entre otras), si bien algunos autores manifiestan recelos en su utilización (Rust, Price y O’Donovan, 2003; Kohn, 2006; Goodrich-Andrade, 2006, Sadler, 2009). Otros estudiosos del tema opinan que la utilidad, fiabilidad y validez de las rúbricas no están definidas con exactitud y que se necesitan más estudios sobre las mismas en diversos contextos educativos para poner de manifiesto su eficacia (Reddy y Andrade, 2010). En este sentido, Turley y Gallagher (2008:87) señalan que "en lugar de declarar todas las rúbricas 'buenas' o 'malas', tenemos que tener en cuenta el contexto en el que se aplican, lo cual ayuda a los educadores a evaluar el valor de las mismas".

Para determinar los niveles de logro en función de los criterios de calidad explicitados, se puede partir de los establecidos por la Taxonomía SOLO (Structured of the Observed Learning Outcomes), basados en el progreso de la incompetencia a la competencia en el aprendizaje (Biggs y Collins, 1982, citados por Hernández Pina. Martínez Clares, Da Fonseca y Rubio, 2005): 
I. Nivel Preestructural: Respuestas centradas en aspectos irrelevantes con contestaciones evasivas del enunciado.

II. Nivel Uniestructural: Respuestas con datos informativos obvios, extraídos directamente del enunciado.

III. Nivel Multiestructural: Respuestas que requieren dos o más informaciones del enunciado, obtenidas directamente de éste y analizadas separadamente, sin relación.

IV. Nivel Relacional: Respuestas extraídas tras analizar el problema, integrando la información en una estructura comprensiva.

V. Nivel de Abstracción: Respuestas que utilizan un principio general y abstracto para ser inferido a otros contextos a partir de un análisis profundo del problema.

Para los autores a los que nos estamos refiriendo, la principal línea divisoria entre estos niveles se encuentra entre el tercero y el cuarto, ya que el objetivo en los tres primeros es responder y reproducir más o menos detalladamente a lo que se pregunta, mientras que en los dos últimos se esperan respuestas estructuradas interrelacionadas y que muestren capacidad de transferencia. En definitiva, se trata de pasar de la mera reproducción a la comprensión.

Respecto al grado de complejidad de las rúbricas, podemos utilizar desde rúbricas sencillas que, en el caso de partir de las competencias a evaluar, integran la formulación de cada una de ellas y los criterios de calidad con tres o cuatro niveles de logro, a rúbricas mucho más complejas, consideradas verdaderas secuencias didácticas (Pimienta, 2011), que suponen la integración de todos los elementos que confluyen en el proceso de enseñanza-aprendizaje, con un número más o menos amplio de niveles de logro. Martínez Rojas (2008) distingue entre la rúbrica global u holística, la cual permite hacer una valoración de conjunto de la competencia evaluada sin determinar criterios de calidad; y la rúbrica analítica, la cual sí necesita formular criterios de calidad previos que ponen de manifiesto la adquisición de la competencia, seguido del establecimiento de los niveles de logro en función de cada criterio. Para la construcción de rúbricas, una herramienta gratuita muy útil disponible en Internet es Rubistar.

Desde nuestro punto de vista, basándonos en las categorías contempladas en las calificaciones universitarias, con el fin de que aportar familiaridad al procedimiento, cuatro niveles de logro pueden resultar adecuados para evaluar el grado de desempeño de una competencia, en relación con el criterio de calidad referido:

1. Suspenso: El estudiante no posee un nivel suficiente de dominio de la competencia.

2. Aprobado: El estudiante desempeña los criterios de calidad de forma aceptable.

3. Notable: El estudiante demuestra un buen dominio de la competencia evaluada.

4. Sobresaliente: El estudiante manifiesta poseer un nivel de competencia excelente.

Como se ha mencionado con anterioridad, en el apartado de evaluación de las guías docentes, el profesorado ha de indicar los instrumentos de recogida de información, con sus correspondientes criterios de valoración y la ponderación otorgada a cada uno de ellos, en función de las competencias a evaluar con cada instrumento. Dicha evaluación puede planificarse y realizarse mediante rúbricas al determinar distintos niveles de logro o desempeño en relación con cada criterio de calidad elegido.

Por ejemplo, supongamos que uno de los instrumentos de evaluación utilizados en una asignatura de Grado en Pedagogía, denominada "Evaluación de programas educativos y 
formativos" (EPEF), es un portafolios discente integrado por parte de las prácticas realizadas dentro de dicha asignatura.

Las competencias transversales $(T)$ que han de adquirir los estudiantes en la asignatura son:

- Ser capaz de expresarse correctamente en su ámbito disciplinar [Transversal 1].

- Ser capaz de gestionar la información y el conocimiento, incluyendo saber utilizar como usuario las herramientas básicas en TIC [Transversal 3].

- Considerar la ética y la integridad intelectual como valores esenciales de la práctica profesional [Transversal 4].

- Ser capaz de trabajar en equipo y de relacionarse con otras personas del mismo o distinto ámbito profesional [Transversal 6].

- Desarrollar habilidades de iniciación a la investigación [Transversal 7].

Las competencias específicas (E) a desarrollar por el alumnado en esta asignatura son:

1. Conocer y utilizar la terminología específica de EPEF.

2. Conocer y aplicar los modelos de evaluación de programas a la práctica educativa.

3. Diseñar proyectos de evaluación de EPEF contextualizados.

4. Adquirir procedimientos para ejecutar EPEF.

5. Interpretar y valorar EPEF realizadas en diferentes contextos.

6. Desarrollar estrategias para realizar informes de EPEF contextualizados.

En la tabla 1 se muestran las competencias que van a ser evaluadas con el portafolios, los criterios de calidad con los que el mismo va a ser valorado y la puntuación máxima que se le puede otorgar - $25 \%$ de la calificación final-. El resto de competencias serán evaluadas con un plan de evaluación y una prueba teórico-práctica.

Tabla 1. Criterios de evaluación del portafolios, competencias a evaluar y puntuación

\begin{tabular}{|c|c|c|c|}
\hline \multicolumn{4}{|c|}{ Portafolios } \\
\hline Con & Eias a & Criterios de calidad & Puntuación \\
\hline$E$ & $\mathrm{~T}$ & & \\
\hline 2 & $\begin{array}{l}1 \\
3 \\
4 \\
6 \\
7\end{array}$ & $\begin{array}{l}\text { 1. Presentación del portafolios. } \\
\text { 2. Dominio de contenidos específicos. } \\
\text { 3. Expresión escrita. } \\
\text { 4. Gestión de la información. } \\
\text { 5. Creatividad y espíritu crítico. } \\
\text { 6. Trabajo grupal y organización del tiempo. }\end{array}$ & $\begin{array}{c}2,5 \\
2,5 * 100=250\end{array}$ \\
\hline
\end{tabular}

Como se aprecia, con el fin de no operar con números excesivamente pequeños, la puntuación total otorgado al instrumento se ha multiplicado por 100, por lo que la valoración máxima del portafolios es de 250 puntos.

A continuación, en la tabla 2, se muestra la rúbrica completa. 
Tabla 2. Rúbrica para evaluar el portafolios

\begin{tabular}{|c|c|c|c|c|c|c|c|}
\hline \multicolumn{8}{|c|}{ Portafolios } \\
\hline \multicolumn{2}{|c|}{$\begin{array}{l}\text { Comp. } \\
\text { evaluadas }\end{array}$} & \multirow{2}{*}{$\begin{array}{c}\text { Criterios } \\
\text { de } \\
\text { calidad }\end{array}$} & \multicolumn{4}{|c|}{ Nivel de logro } & \multirow{2}{*}{ Puntos } \\
\hline $\mathrm{E}$ & $\mathbf{T}$ & & Sobresaliente & Notable & Aprobado & Suspenso & \\
\hline & 4 & 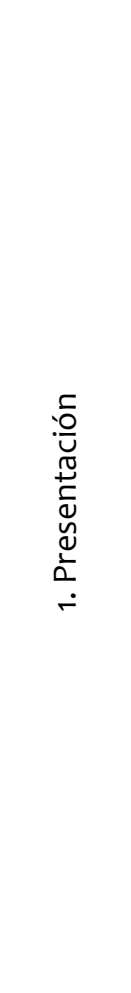 & $\begin{array}{c}\text { Cada práctica } \\
\text { incluye todos } \\
\text { los } \\
\text { elementos } \\
\text { obligatorios } \\
\text { descritos en } \\
\text { la guía } \\
\text { docente de la } \\
\text { asignatura. } \\
\text { Se presentan } \\
\text { con absoluta } \\
\text { limpieza, la } \\
\text { letra es muy } \\
\text { legible, los } \\
\text { márgenes y } \\
\text { espacios } \\
\text { entre } \\
\text { elementos y } \\
\text { párrafos son } \\
\text { precisos y el } \\
\text { papel está } \\
\text { muy bien } \\
\text { aprovechado } \\
\text {. }\end{array}$ & $\begin{array}{c}\text { Cada práctica } \\
\text { incluye todos } \\
\text { los } \\
\text { elementos } \\
\text { obligatorios } \\
\text { descritos en } \\
\text { la guía } \\
\text { docente de la } \\
\text { asignatura. } \\
\text { Hay uno o } \\
\text { dos tachones } \\
\text { leves, la letra } \\
\text { es bastante } \\
\text { legible, los } \\
\text { márgenes y } \\
\text { espacios son } \\
\text { medianamen } \\
\text { te precisos y } \\
\text { el papel está } \\
\text { bien } \\
\text { aprovechado } \\
\text {. }\end{array}$ & $\begin{array}{c}\text { Falta incluir } \\
\text { en cada } \\
\text { práctica uno o } \\
\text { dos } \\
\text { elementos } \\
\text { obligatorios } \\
\text { no relevantes } \\
\text { (ni los } \\
\text { componentes } \\
\text { del grupo, ni } \\
\text { la ejecución, } \\
\text { ni la } \\
\text { evaluación). } \\
\text { Hay tres } \\
\text { tachones } \\
\text { como } \\
\text { máximo, la } \\
\text { letra es } \\
\text { medianament } \\
\text { e legible, los } \\
\text { márgenes y } \\
\text { espacios son } \\
\text { poco precisos } \\
\text { y el papel } \\
\text { escasamente } \\
\text { aprovechado. }\end{array}$ & $\begin{array}{l}\text { Falta incluir } \\
\text { más de dos } \\
\text { elementos } \\
\text { obligatorios } \\
\text { en cada } \\
\text { práctica. Se } \\
\text { aprecian más } \\
\text { de tres } \\
\text { tachones, la } \\
\text { letra es } \\
\text { ilegible, los } \\
\text { márgenes y } \\
\text { espacios son } \\
\text { muy poco o } \\
\text { nada } \\
\text { precisos y el } \\
\text { papel no se } \\
\text { aprovecha. } \\
\text { Se comete } \\
\text { plagio total o } \\
\text { parcial. }\end{array}$ & $\begin{array}{c}6 \% \\
\text { (15 p.) }\end{array}$ \\
\hline 4 & 7 & 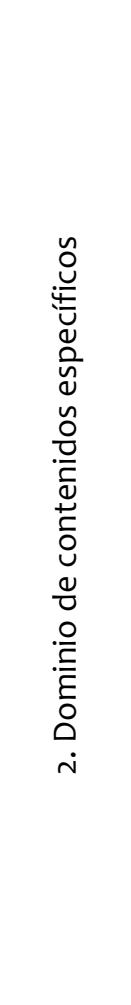 & $\begin{array}{l}\text { Las prácticas } \\
\text { son realizadas } \\
\text { con total } \\
\text { dominio y } \\
\text { precisión de su } \\
\text { contenido, se } \\
\text { utiliza } \\
\text { correctamente } \\
\text { toda la } \\
\text { terminología, } \\
\text { las ideas están } \\
\text { muy bien } \\
\text { fundamenta- } \\
\text { das y se ha } \\
\text { realizado de } \\
\text { acuerdo con } \\
\text { los reque- } \\
\text { rimientos de } \\
\text { ejecución de las } \\
\text { mismas } \\
\text { explicitados en } \\
\text { la guía docente } \\
\text { y explicados en } \\
\text { clase. }\end{array}$ & $\begin{array}{l}\text { Las prácticas } \\
\text { son realizadas } \\
\text { con utilización } \\
\text { correcta de la } \\
\text { mayor parte de } \\
\text { la terminología. } \\
\text { Aunque con } \\
\text { dominio de su } \\
\text { contenido y } \\
\text { fundamentan- } \\
\text { do las ideas, } \\
\text { necesitan } \\
\text { ampliar o } \\
\text { corregir uno o } \\
\text { dos aspectos } \\
\text { poco } \\
\text { relevantes, de } \\
\text { acuerdo con } \\
\text { los reque- } \\
\text { rimientos de } \\
\text { ejecución de las } \\
\text { mismas. }\end{array}$ & $\begin{array}{l}\text { Las prácticas } \\
\text { son realizadas } \\
\text { con moderado } \\
\text { dominio de su } \\
\text { contenido y } \\
\text { solo parte de la } \\
\text { terminología } \\
\text { utilizada es } \\
\text { correcta. Se } \\
\text { fundamentan } \\
\text { de forma } \\
\text { suficiente las } \\
\text { ideas, pero se } \\
\text { necesita } \\
\text { ampliar o } \\
\text { corregir un } \\
\text { aspecto } \\
\text { relevante o tres } \\
\text { o cuatro poco } \\
\text { relevantes, de } \\
\text { acuerdo con los } \\
\text { requerimien- } \\
\text { tos de } \\
\text { ejecución de las } \\
\text { mismas. }\end{array}$ & $\begin{array}{l}\text { No se aprecia } \\
\text { dominio del } \\
\text { contenido de } \\
\text { las prácticas. La } \\
\text { terminología es } \\
\text { incorrecta y las } \\
\text { ideas se } \\
\text { fundamentan } \\
\text { muy poco o } \\
\text { nada. Es } \\
\text { necesario } \\
\text { ampliar o } \\
\text { corregir más de } \\
\text { cuatro } \\
\text { aspectos poco } \\
\text { relevantes o } \\
\text { más de dos } \\
\text { relevantes, de } \\
\text { acuerdo con los } \\
\text { requerimien- } \\
\text { tos de } \\
\text { ejecución de las } \\
\text { mismas. } \\
\text { Se comete } \\
\text { plagio total o } \\
\text { parcial. }\end{array}$ & $\begin{array}{c}70 \% \\
(175 \text { p.) }\end{array}$ \\
\hline
\end{tabular}




\begin{tabular}{|c|c|c|c|c|c|c|c|}
\hline \multicolumn{8}{|c|}{ Portafolios } \\
\hline \multicolumn{2}{|c|}{$\begin{array}{l}\text { Comp. } \\
\text { evaluadas }\end{array}$} & \multirow{2}{*}{$\begin{array}{l}\text { Criterios } \\
\text { de calidad }\end{array}$} & \multicolumn{4}{|c|}{ Nivel de logro } & \multirow{2}{*}{ Puntos } \\
\hline $\mathbf{E}$ & $\mathbf{T}$ & & Sobresaliente & Notable & Aprobado & Suspenso & \\
\hline & $\begin{array}{l}1 \\
4\end{array}$ & 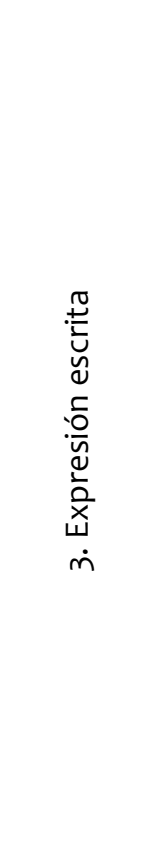 & $\begin{array}{l}\text { Las prácticas } \\
\text { se presentan } \\
\text { muy bien } \\
\text { estructuradas, } \\
\text { con alta } \\
\text { claridad } \\
\text { expositiva, } \\
\text { gran dominio } \\
\text { del lenguaje y } \\
\text { utilización de } \\
\text { vocabulario } \\
\text { técnico y } \\
\text { preciso. Existe } \\
\text { elevada } \\
\text { capacidad de } \\
\text { análisis y } \\
\text { síntesis, así } \\
\text { como } \\
\text { corrección } \\
\text { ortográfica y } \\
\text { gramatical. }\end{array}$ & $\begin{array}{l}\text { Las prácticas } \\
\text { están } \\
\text { bastante } \\
\text { estructuradas, } \\
\text { con suficiente } \\
\text { claridad } \\
\text { expositiva, } \\
\text { dominio del } \\
\text { lenguaje y, a } \\
\text { menudo, } \\
\text { utilización de } \\
\text { vocabulario } \\
\text { técnico y } \\
\text { preciso. Existe } \\
\text { bastante } \\
\text { capacidad de } \\
\text { análisis y } \\
\text { síntesis, así } \\
\text { como } \\
\text { corrección } \\
\text { ortográfica y } \\
\text { gramatical. }\end{array}$ & $\begin{array}{l}\text { Las prácticas } \\
\text { están poco } \\
\text { estructuradas, } \\
\text { con moderada } \\
\text { claridad } \\
\text { expositiva, } \\
\text { poco dominio } \\
\text { del lenguaje, y } \\
\text { vocabulario } \\
\text { técnico y } \\
\text { preciso. Se } \\
\text { aprecia } \\
\text { suficiente } \\
\text { capacidad de } \\
\text { análisis y } \\
\text { síntesis y hay } \\
\text { una o dos } \\
\text { incorrecciones } \\
\text { ortográficas o } \\
\text { gramaticales, } \\
\text { poco } \\
\text { relevantes. }\end{array}$ & $\begin{array}{l}\text { Las prácticas } \\
\text { carecen de } \\
\text { estructuración } \\
\text {, no se domina } \\
\text { el lenguaje y el } \\
\text { vocabulario es } \\
\text { poco técnico y } \\
\text { preciso. Hay } \\
\text { escasa } \\
\text { capacidad de } \\
\text { análisis y } \\
\text { síntesis y más } \\
\text { de dos } \\
\text { incorrecciones } \\
\text { ortográficas o } \\
\text { gramaticales. } \\
\text { Se comete } \\
\text { plagio total o } \\
\text { parcial. }\end{array}$ & $\begin{array}{c}7 \% \\
(17,5 \text { p.) }\end{array}$ \\
\hline & $\begin{array}{l}4 \\
7\end{array}$ & 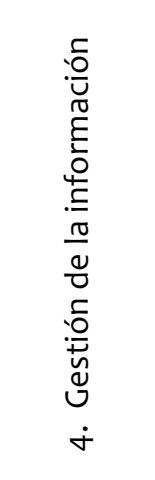 & $\begin{array}{l}\text { Todas las citas } \\
\text { en el texto y las } \\
\text { referencias } \\
\text { bibliográficas } \\
\text { son muy } \\
\text { relevantes, } \\
\text { actuales y según } \\
\text { la APA. Se } \\
\text { utilizan mucho } \\
\text { las TIC. }\end{array}$ & $\begin{array}{l}\text { La mayoría de } \\
\text { las citas en el } \\
\text { texto y las } \\
\text { referencias } \\
\text { bibliográficas } \\
\text { son relevantes, } \\
\text { actuales y, } \\
\text { según la APA. Se } \\
\text { utilizan bastante } \\
\text { las TIC. }\end{array}$ & $\begin{array}{c}\text { Solo algunas } \\
\text { citas en el texto } \\
\text { y las referencias } \\
\text { bibliográficas } \\
\text { son relevantes, } \\
\text { actuales y según } \\
\text { la APA. Se } \\
\text { utilizan } \\
\text { medianamen-te } \\
\text { las TIC. }\end{array}$ & $\begin{array}{l}\text { Ninguna o casi } \\
\text { ninguna de las } \\
\text { citas y } \\
\text { referencias son } \\
\text { actuales, ni } \\
\text { relevantes, ni } \\
\text { siguen la } \\
\text { normativa APA. } \\
\text { Se utilizan poco } \\
\text { o nada las TIC. } \\
\text { Se comete plagio } \\
\text { total o parcial. }\end{array}$ & $\begin{array}{c}5 \% \\
\text { (12,5 p.) }\end{array}$ \\
\hline & $\begin{array}{l}4 \\
7\end{array}$ & 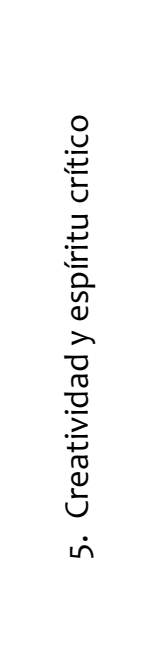 & $\begin{array}{c}\text { Las prácticas } \\
\text { están realizadas } \\
\text { con mucha } \\
\text { creatividad e } \\
\text { introducen altas } \\
\text { cotas de } \\
\text { innovación que } \\
\text { las hacen muy } \\
\text { originales. Son } \\
\text { abordadas con } \\
\text { alto espíritu } \\
\text { crítico } \\
\text { constructivo, sin } \\
\text { dejar de partir } \\
\text { de fundamenta- } \\
\text { ción teórica y } \\
\text { metodológica. }\end{array}$ & $\begin{array}{l}\text { Las prácticas } \\
\text { están realizadas } \\
\text { con bastante } \\
\text { creatividad e } \\
\text { introducen } \\
\text { varios detalles } \\
\text { innovadores que } \\
\text { las hacen } \\
\text { originales. Son } \\
\text { abordadas con } \\
\text { espíritu crítico } \\
\text { constructivo, sin } \\
\text { dejar de partir } \\
\text { de fundamenta- } \\
\text { ción teórica y } \\
\text { metodológica. }\end{array}$ & $\begin{array}{l}\text { Las prácticas } \\
\text { están realizadas } \\
\text { con suficiente } \\
\text { creatividad e } \\
\text { introducen } \\
\text { algunos detalles } \\
\text { innovadores que } \\
\text { las hacen algo } \\
\text { originales. Son } \\
\text { abordadas con } \\
\text { algo de espíritu } \\
\text { constructivo, sin } \\
\text { dejar de partir } \\
\text { de una } \\
\text { fundamentación } \\
\text { teórica y } \\
\text { metodológica. }\end{array}$ & $\begin{array}{l}\text { Las prácticas } \\
\text { carecen de } \\
\text { creatividad, no } \\
\text { poseen detalles } \\
\text { innovadores y, } \\
\text { por lo tanto, no } \\
\text { son nada } \\
\text { originales. Se } \\
\text { limitan a } \\
\text { reproducir lo } \\
\text { explicado en } \\
\text { clase. No se } \\
\text { aprecia espíritu } \\
\text { crítico o éste es } \\
\text { destructivo. } \\
\text { Se comete plagio } \\
\text { total o parcial. }\end{array}$ & $\begin{array}{c}5 \% \\
(12,5 \text { p.) }\end{array}$ \\
\hline
\end{tabular}




\begin{tabular}{|c|c|c|c|c|c|c|c|}
\hline \multicolumn{8}{|c|}{ Portafolios } \\
\hline \multicolumn{2}{|c|}{$\begin{array}{c}\text { Comp. } \\
\text { evaluadas }\end{array}$} & \multirow{2}{*}{$\begin{array}{l}\text { Criterios } \\
\text { de calidad }\end{array}$} & \multicolumn{4}{|c|}{ Nivel de logro } & \multirow{2}{*}{ Puntos } \\
\hline $\mathrm{E}$ & $\mathbf{T}$ & & Sobresaliente & Notable & Aprobado & Suspenso & \\
\hline & $\begin{array}{l}4 \\
6\end{array}$ & 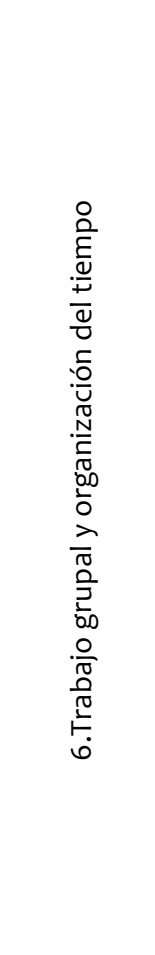 & $\begin{array}{l}\text { En general, los } \\
\text { miembros del } \\
\text { grupo se han } \\
\text { implicado } \\
\text { perfectamente } \\
\text { en todas las } \\
\text { prácticas, } \\
\text { trabajando } \\
\text { mediante } \\
\text { aprendizaje } \\
\text { cooperativo, de } \\
\text { acuerdo con los } \\
\text { criterios } \\
\text { establecidos } \\
\text { para la } \\
\text { evaluación } \\
\text { individual. Las } \\
\text { distintas } \\
\text { prácticas se han } \\
\text { entregado } \\
\text { siempre en las } \\
\text { fechas } \\
\text { señaladas. }\end{array}$ & $\begin{array}{l}\text { En general, los } \\
\text { miembros del } \\
\text { grupo se han } \\
\text { implicado bien } \\
\text { en la mayoría de } \\
\text { las prácticas, } \\
\text { trabajando } \\
\text { mediante } \\
\text { aprendizaje } \\
\text { cooperativo, de } \\
\text { acuerdo con casi } \\
\text { todos los } \\
\text { criterios } \\
\text { establecidos } \\
\text { para la } \\
\text { evaluación } \\
\text { individual. En } \\
\text { una o dos } \\
\text { ocasiones, la } \\
\text { práctica } \\
\text { requerida no se } \\
\text { ha entregado en } \\
\text { las fechas } \\
\text { señaladas. }\end{array}$ & $\begin{array}{l}\text { En general, los } \\
\text { miembros del } \\
\text { grupo se han } \\
\text { implicado } \\
\text { moderadamente } \\
\text { en parte de las } \\
\text { prácticas, } \\
\text { trabajando } \\
\text { mediante } \\
\text { aprendizaje } \\
\text { cooperativo, de } \\
\text { acuerdo con } \\
\text { algunos de los } \\
\text { criterios } \\
\text { establecidos } \\
\text { para la } \\
\text { evaluación } \\
\text { individual. En } \\
\text { tres o cuatro } \\
\text { ocasiones, la } \\
\text { práctica } \\
\text { requerida no se } \\
\text { ha entregado en } \\
\text { las fechas } \\
\text { señaladas. }\end{array}$ & $\begin{array}{c}\text { En general, los } \\
\text { miembros del } \\
\text { grupo no se han } \\
\text { implicado en las } \\
\text { prácticas, no han } \\
\text { trabajado } \\
\text { mediante } \\
\text { aprendizaje } \\
\text { cooperativo, en } \\
\text { función de los } \\
\text { criterios } \\
\text { establecidos } \\
\text { para la } \\
\text { evaluación } \\
\text { individual. En } \\
\text { más de cuatro } \\
\text { ocasiones, la } \\
\text { práctica } \\
\text { requerida no se } \\
\text { ha entregado en } \\
\text { las fechas } \\
\text { señaladas. } \\
\text { Se comete plagio } \\
\text { total o parcial. }\end{array}$ & $\begin{array}{c}7 \% \\
\text { (17,5 p.) }\end{array}$ \\
\hline \multicolumn{3}{|c|}{ Puntuación } & $225-250$ & $175-224$ & $125-174$ & $0-124$ & 250 \\
\hline
\end{tabular}

La última fila de la tabla 2 indica el intervalo de puntos que, globalmente, se corresponde con cada uno de los distintos niveles de logro y, que como se ha mencionado más arriba, se refiere a las categorías contempladas actualmente en la evaluación de los estudiantes universitarios -suspenso, aprobado, notable y sobresaliente-, considerando que el 10 supone la matrícula de honor, siempre que el número de dieces no supere el $5 \%$ del alumnado.

Con el fin de precisar la puntuación a otorgar a cada uno de los indicadores de evaluación que integran los respectivos criterios, en las tablas que siguen $-3,4,5,6,7$ y 8 - se muestra el valor numérico máximo correspondiente a cada uno de dichos indicadores. Los estudiantes, continuando con la modalidad de aprendizaje cooperativo, deberán consensuar la valoración concedida a cada indicador a partir del trabajo realizado, la cual será contrastada posteriormente con la otorgada por el profesorado. Esta puntuación del docente, al tomar como referente de evaluación la misma rúbrica, no debe alejarse de la que el alumnado ha establecido mediante procedimientos de autoevaluación. En caso de que ambas valoraciones se encuentren alejadas, el profesor puede tener una entrevista con los estudiantes para que éstos expliquen detalladamente, cómo han procedido a autoevaluar su portafolios.

Asimismo, en dichas tablas, también se incluye, a título informativo, la calificación que se obtendría en función de la puntuación global obtenida en cada criterio.

Seguidamente, se muestran las tablas mencionadas. 
Tabla 3. Valoración de los indicadores del criterio presentación

\begin{tabular}{|c|c|c|c|}
\hline \multicolumn{4}{|c|}{ 1- PRESENTACIÓN } \\
\hline \multicolumn{2}{|c|}{ Indicador } & Puntuación máxima & Puntuación otorgada \\
\hline $\begin{array}{l}\text { 1. Inclusión de elementos } \\
\mathrm{N}^{\circ} \text { práctica y título } \\
\mathrm{N}^{\circ} \text { grupo y componentes } \\
\text { Enunciado } \\
\text { Ejecución } \\
\text { Referencias } \\
\text { Competencias } \\
\text { Evaluación grupal } \\
\text { Evaluación individual }\end{array}$ & $\begin{array}{r}\mathrm{SI} / \mathrm{NO} \\
\mathrm{SI} / \mathrm{NO} \\
\mathrm{SI} / \mathrm{NO} \\
2 \text { (SI/NO) } \\
\mathrm{SI} / \mathrm{NO} \\
\mathrm{SI} / \mathrm{NO} \\
\mathrm{SI} / \mathrm{NO} \\
\mathrm{SI} / \mathrm{NO} \\
\end{array}$ & 9 & \\
\hline \multicolumn{2}{|l|}{ 2. Limpieza } & 2 & \\
\hline \multicolumn{2}{|l|}{ 3. Legibilidad letra } & 2 & \\
\hline \multicolumn{2}{|c|}{ 4. Márgenes, espacios y aprovechamiento del papel } & 2 & \\
\hline \multicolumn{2}{|l|}{ SUBTOTAL } & 15 & \\
\hline \multicolumn{4}{|c|}{ Calificación } \\
\hline \multicolumn{2}{|c|}{$0-7,49$} & \multicolumn{2}{|c|}{ Suspenso } \\
\hline \multicolumn{2}{|c|}{$7,5-10,49$} & \multicolumn{2}{|c|}{ Aprobado } \\
\hline \multicolumn{2}{|c|}{$10,5-13,49$} & \multicolumn{2}{|c|}{ Notable } \\
\hline \multicolumn{2}{|c|}{$13,5-15$} & \multicolumn{2}{|c|}{ Sobresaliente } \\
\hline
\end{tabular}

Tabla 4. Valoración de los indicadores del criterio dominio de contenidos específicos

\begin{tabular}{|l|c|c|}
\hline \multicolumn{3}{|c|}{ 2- DOMINIO DE CONTENIDOS ESPECífICOS } \\
\hline Indicador & Puntuación máxima & Puntuación otorgada \\
\hline 1. Precisión del contenido en la ejecución & $\mathbf{5 0}$ & \\
\hline 2. Utilización de terminología específica & $\mathbf{5 0}$ & \\
\hline 3. Fundamentación de ideas & $\mathbf{7 5}$ & \\
\hline SUBTOTAL Calificación & $\mathbf{1 7 5}$ \\
\hline \multicolumn{1}{|c|}{ Suspenso } \\
\hline $0-87,49$ & Aprobado \\
\hline $87,5-122,49$ & Sotable \\
\hline $122,5-157,49$ & Sobresaliente \\
\hline $157,5-175$ &
\end{tabular}

Tabla 5. Valoración de los indicadores del criterio expresión escrita

\begin{tabular}{|c|c|c|}
\hline \multicolumn{3}{|c|}{ 3- EXPRESIÓN ESCRITA } \\
\hline Indicador & Puntuación máxima & Puntuación otorgada \\
\hline 1. Estructuración de elementos & 3 & \\
\hline 2. Claridad expositiva & 3 & \\
\hline 3. Amplitud y precisión de vocabulario & 3 & \\
\hline 4. Capacidad de análisis y síntesis & 3 & \\
\hline 5. Corrección ortográfica y gramatical & 5,5 & \\
\hline SUBTOTAL & 17,5 & \\
\hline \multicolumn{3}{|c|}{ Calificación } \\
\hline $0-8,74$ & \multicolumn{2}{|c|}{ Suspenso } \\
\hline $8,75-12,24$ & \multicolumn{2}{|c|}{ Aprobado } \\
\hline $12,25-15,74$ & \multicolumn{2}{|c|}{ Notable } \\
\hline $15,75-17,5$ & \multicolumn{2}{|c|}{ Sobresaliente } \\
\hline
\end{tabular}


Tabla 6. Valoración de los indicadores del criterio gestión de la información

\begin{tabular}{|l|c|c|}
\hline \multicolumn{3}{|c|}{ 4- GESTIÓN DE LA INFORMACIÓN } \\
\hline \multicolumn{1}{|c|}{ Indicador } & Puntuación máxima & Puntuación otorgada \\
\hline 1. Suficiencia y relevancia de fuentes consultadas & $\mathbf{4}$ & \\
\hline 2. Utilización de TIC & 3,5 & \\
\hline 3. Adecuación de citas y referencias (APA) & $\mathbf{1 2 , 5}$ & \\
\hline SUBTOTAL Calificación & \multicolumn{2}{c|}{ Suspenso } \\
\hline \multicolumn{1}{|c|}{ Aprobado } \\
\hline $0-6,24$ & \multicolumn{2}{c|}{ Sotable } \\
\hline $6,25-8,74$ & \multicolumn{2}{c|}{ Sobresaliente } \\
\hline $11,75-11,24$ &
\end{tabular}

Tabla 7. Valoración de los indicadores del criterio creatividad y espíritu crítico

\begin{tabular}{|l|c|c|}
\hline \multicolumn{3}{|c|}{ 5- CREATIVIDAD Y ESPÍRITU CRÍTICO } \\
\hline \multicolumn{1}{|c|}{ Indicador } & Puntuación máxima & Puntuación otorgada \\
\hline 1. Creatividad e innovación & 7 & \\
\hline 2. Espíritu crítico constructivo & 5,5 & \\
\hline SUBTOTAL & $\mathbf{1 2 , 5}$ & \\
\hline \multicolumn{1}{|c|}{ Calificación } \\
\hline $0-6,24$ & Suspenso \\
\hline $6,25-8,74$ & Aprobado \\
\hline $8,75-11,24$ & Notable \\
\hline $11,25-12,5$ & \multicolumn{2}{c|}{ Sobresaliente } \\
\hline
\end{tabular}

Tabla 8. Valoración de los indicadores del criterio trabajo grupal y organización del tiempo

\begin{tabular}{|l|c|c|}
\hline \multicolumn{2}{|c|}{ 6- TRABAJO GRUPAL Y ORGANIZACIÓN DEL TIEMPO } \\
\hline \multicolumn{1}{|c|}{ Indicador } & Puntuación máxima & Puntuación otorgada \\
\hline 1. Aprendizaje cooperativo & $\mathbf{9 , 5}$ & \\
\hline 2. Entregas en fechas indicadas & $\mathbf{8}$ & \\
\hline SUBTOTAL & $\mathbf{1 7 , 5}$ \\
\hline & Calificación & Suspenso \\
\hline $0-8,74$ & \multicolumn{2}{c|}{ Aprobado } \\
\hline $8,75-12,24$ & \multicolumn{2}{c|}{ Sobresable } \\
\hline $12,25-15,74$ & \multicolumn{2}{c}{} \\
\hline $15,75-17,5$ & \multicolumn{2}{c|}{} \\
\hline
\end{tabular}

Tal y como se explicita en la rúbrica contemplada en la tabla 2, en caso de cometer plagio parcial o total el portafolios está suspenso, por lo que a las tablas que preceden, se puede añadir la siguiente información:

\begin{tabular}{|l|l|}
\hline SE COMETE PLAGIO PARCIAL O TOTAL & SI * NO \\
\hline
\end{tabular}

* En este caso, el portafolios está suspenso 
La puntuación en cada práctica se calcula mediante la aplicación de la siguiente fórmula:

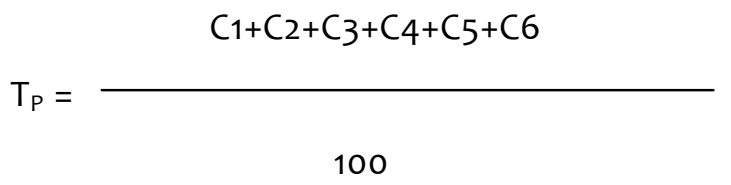

Ci: Puntuación obtenida por el grupo en cada criterio

La calificación global del portafolios se obtiene calculando la media aritmética o ponderada -según proceda- de todas las prácticas que lo integran.

\section{Conclusiones}

En el nuevo modelo educativo de formación por competencias en educación superior todos los elementos del curriculum giran en torno al desarrollo de las mismas por parte del estudiante, originando que éste pase a ser el verdadero protagonista en la construcción de sus aprendizajes.

La evaluación, como elemento clave en este nuevo modelo competencial defendido por el EEES, ha de estar orientada, pues, a demostrar el logro de competencias, tanto específicas como transversales. Por lo tanto, los contenidos ya no constituyen el objeto de la evaluación, sino que éstos -tanto teóricos como prácticos- se convierten en las herramientas para el desarrollo de las competencias. Con ello, el problema estriba en que la evaluación se sitúa en los espacios donde la mirada del profesorado universitario y de los propios estudiantes, habitualmente no ve nada porque no está acostumbrada a mirar, por lo que se necesitan recursos, formación y tiempo para que la lente humana pueda adaptarse a ello y obrar en consecuencia.

Por otra parte, en el modelo educativo universitario tradicional, los contenidos y las actividades son presencias, mientras que la evaluación se viene resolviendo en las ausencias. En el modelo competencial, para que la evaluación de los aprendizajes del alumnado se convierta en una auténtica herramienta formativa para los estudiantes, éstos deben participar activamente en los procesos evaluativos mediante estrategias de autoevaluación y evaluación recíproca. De esta forma, dicha evaluación no será únicamente del aprendizaje, sino fundamentalmente para el aprendizaje.

Los procedimientos de evaluación universitarios deben incluir variedad de instrumentos de recogida de información capaces de poner de manifiesto el logro de múltiples competencias a adquirir por los estudiantes. Dichos instrumentos, asociados a una o varias competencias, han de estar centrados en la construcción de la respuesta, no en la mera reproducción de la misma. Igualmente, se deben establecer los criterios de calidad para cada instrumento con sus diferentes niveles de logro, a través de la construcción de rúbricas que ayuden a realizar una evaluación más objetiva, justa y transparente. Cada instrumento de recogida de información ha de incluir una ponderación específica, de manera que los estudiantes sepan, no solo en base a qué se les evalúa, sino también el peso de cada procedimiento en función de las competencias adquiridas. De esta forma, la información será fácilmente interpretable para profesores y estudiantes en un escenario de participación activa de todos los colectivos implicados en los procesos educativos. 


\section{Bibliografía}

Álvarez, I. (2008). Evaluación del aprendizaje: una Mirada retrospectiva y prospectiva desde la divulgación científica. Revista Electrónica de Investigación Psicoeducativa, 14 (1), 235-272

Andrade, H. y Du, Y. (2005). Student Perspectives on Rubric-referenced Assessment. Practical Assessment, Research and Evaluation, 10 (3), 1-11.

Baartman, L.K.J. y Braun, E. (2011). Editorial. Assessment \& Evaluation in Higher Education, 36 (4), $377-380$

Baartman, L.K.J., Bastiaens, T.J., Kirschner, P.A. y Van der Vleuten, C.P.M. (2007). Evaluating assessment quality in competence-based education: A qualitative comparison of two frameworks. Educational Research Review, 2 (2), 114-129.

Biggs, J.B. (1999). Teaching for quality learning at university. Buckingham: Open University Press.

Biggs, J.B. (2004). Teaching for Quality Learning at University. Madrid: Narcea.

Biggs, J.B. y Collins, K.F. (1982). Evaluating the Quality of Learning: the SOLO Taxonomy. New York: Academic Press.

Black, P. y William, D. (1998). Assessment and classroom learning. Assessment in Education, 5 (1), 7-74.

Boud, D. y Falchikov, N. (2006). Aligning Assessment with Long-Term Learning. Assessment \& Evaluation in Higher Education, 31 (4), 399-413.

Bretones, A. (2008). Participación del alumnado de Educación Superior en su evaluación. Revista de Educación, 347, 181-202.

Brough, J.A. y Pool, J.E. (2005). Integrating learning and assessment: The development of an assessment culture. In J. Etim (Ed.) Curriculum integration K-12: Theory and practice (pp. 196-204). Lanham, MD: University Press of America.

Campbell, A. (2005). Application of ICT and rubrics to the assessment process where professional judgement is involved: the features of an e-marking tool. Assessment \& Evaluation in Higher Education, 30 (5), 529-37.

Castro, M. (2010). ¿Qué sabemos de la medida de las competencias? Características y problemas psicométricos en la evaluación de competencias, Bordón, 63 (1), 109-123.

Colás, P., Jiménez, R. y Villaciervos, P. (2005). Portafolios y desarrollo de competencias profesionales en el marco del Espacio Europeo de Educación Superior. Revista de Ciencias de la Educación, 204, 519-538.

Cowan, J. (2010). Developing the ability for making evaluative judgements. Teaching In Higher Education, 15 (3), 323-334.

Dochy, F., Segers, M. y Sluijsmans, D. (1999). The Use of Self-, Peer and Co-Assessment in Higher Education: a Review. Studies in Higher Education, 24 (3), 331-350.

Draper, J. (2012). Choosing teaching in Hong Kong: a strategy to survive the financial crisis? Educational Research, 54 (2), 199-211.

Dunbar, N.E., Brooks C. y Kubicka-Miller, T. (2006). Oral Communication Skills in Higher Education: Using a Performance-Based Evaluation Rubric to Assess Communication Skills. Innovative Higher Education 31 (2), 116-28. 
Echeverría, B. (2002). Gestión de la competencia de acción profesional. Revista de Investigación Educativa, 20 (1), 7-43.

Falchikov. N. (2005). Improving Assessment Through Student Involvement. Practical solutions for aiding learning in higher and further education. London: RoutledgeFalmer.

Frost, J., De Pont, G. y Brailsford, I. (2012). Expanding assessment methods and moments in history. Assessment \& Evaluation In Higher Education, 37 (3), 293-304.

Gairín, J. et al (2009). Guía para la evaluación de competencias en el Área de Ciencias Sociales. Barcelona: Agència per a la Qualitat del Sistema Universitari de Catalunya. Consultado el 5 de marzo de 2012 en http://www.aqu.cat/doc/doc_14646947_1.pdf.

Galán, A. (2007). El perfil del profesor universitario. Situación actual y retos de futuro. Madrid: Ediciones Encuentro.

García Sanz, M.P. (2008). Guías docentes de asignaturas de Grado en el EEES. Orientaciones para su elaboración. Murcia: Editum.

García Sanz, M.P. (2011). Innovaciones orientadas al EEES. Las competencias y su evaluación como elementos de planificación en el marco del EEES. En J.J. Maquilón (coord.). La formación del profesorado en el siglo XXI. Propuestas ante los cambios económicos, sociales y culturales. Murcia: Editum.

García Sanz, M.P. (2012). Fundamentos teóricos y metodológicos de la evaluación de programas. Murcia: D.M.

Gerard, F.M. y BIEF (2008). Évaluer des compétences. Guide pratique. Bruxelles: De Boeck.

Gibbs, G. (1999) Using assessment strategically to change the way students learn. In S. Brown y A. Glasner (Eds) Assessment Matters in Higher Education: Choosing and Using Diversity Approaches (pp. 41-53). Buckingham: Open University Press.

Gijbels, D. (2011). Assessment of vocational competence in higher education: reflections and prospects. Assessment \& Evaluation in Higher Education, 36 (4), 381-383.

Goodrich-Andrade, H. (2006). The trouble with a narrow view of rubrics. English Journal, 95 (6), 9 .

Guilles, J.L., Detroz, P. y Blais, J.G. (2011). An international online survey of the practices and perceptions of higher education professors with respect to the assessment of learning in the classroom. Assessment \& Evaluation In Higher Education, 36 (6), 719733.

Hafner, J.C. y Hafner P.M. (2003). Quantitative analysis of the rubric as an assessment tool: an empirical study of student peer-group rating. International Journal of Science Education 25 (12), 1509-28.

Harman, K. y McDowell, L. (2011). Assessment talk in Design: the multiple purposes of assessment in HE. Teaching In Higher Education, 16 (1), 41-52.

Hernández Pina, F., Martínez Clares, P., Da Fonseca Rosario, P. y Rubio Espín, M. (2005). Aprendizaje, competencias y rendimiento en Educación Superior. Madrid: La Muralla.

Hernández, R. (2012). Does continuous assessment in higher education support student learning? Higher Education, 64 (4), 489-502.

Holmes, L. (2001). Reconsidering graduate employability: The "graduate identity" approach. Quality in Higher Education, 7 (2), 111-119. 
Huertas, J.A. (2009). Aprender a fijarse metas: nuevos estilos motivacionales. En J.I. Pozo y M.P. Pérez (coords.). Psicología del aprendizaje universitario: la formación en competencias (pp. 164-181). Madrid: Morata.

Ibarra, M.S., Rodríguez, G. y Gómez, M.A. (2012). La evaluación entre iguales: beneficios y estrategias para su práctica en la universidad. Revista de Educación, 359, 206-231.

Imbernón, F. (2006). La profesión docente desde el punto de vista internacional ¿qué dicen los informes? Revista de Educación, 340, 41-49.

Inda, S., Álvarez, S. y Álvarez, R. (2008). Métodos de evaluación en la enseñanza superior. Revista de Investigación Educativa, 26 (2), 539-552.

Jonsson, A. y Svingby, G. (2007). The use of scoring rubrics: Reliability, validity and educational consequences. Educational Research Review, 2 (2), 130-144.

King, R., Marginson, S. y Naidoo, R (eds.). (2011). Handbook on globalization and higher education. Northampton, MA, USA: Edward Elgar publishing.

Kohn, A. (2006). The Trouble with Rubrics. The English Journal. 95 (4), 12-15.

Lemanski, C. (2011). Access and assessment? Incentives for independent study. Assessment \& Evaluation in Higher Education, 36 (5), 565-581.

Livingston, K. (2012) Approaches to professional development of teachers in Scotland: pedagogical innovation or financial necessity? Educational Research, 54 (2), 161-172.

López Pastor, V. (2009). Fundamentación teórica y revisión del estado de la cuestión. En V. López Pastor (coord.). Evaluación formativa y compartida en Educación Superior (pp. 45-64). Madrid: Narcea.

López Ruiz, J.I. (2011). Un giro copernicano en la enseñanza universitaria: formación por competencias, Revista de Educación, 356, 279-301.

López Ruiz, J.I. (2011). Un giro copernicano en la enseñanza universitaria: formación por competencias, Revista de Educación, 356, 279-301.

Mansilla, V.B., Duraisingh, E.D., Wolfe, C.R. y Haynes, C. (2009). Targeted Assessment Rubric: An Empirically Grounded Rubric for Interdisciplinary Writing. The Journal of Higher Education, 80 (3), 334-353.

Martín del Pozo, R.; Fernández-Lozano, P., González-Ballesteros, M. y De Juanas, A. (2013). El dominio de los contenidos escolares: competencia profesional y formación inicial de maestros. Revista de Educación, 360, 363-387.

Martínez Clares, P. y Echeverría, B. (2009). Formación basada en competencias. Revista de Investigación Educativa, 29 (1), 125-147.

Martínez Rojas, J.G. (2008). Las rúbricas en la evaluación escolar: Su construcción y su uso. Avances en Medición, 6, 129-138. Consultado el 21 de febrero de 2012 en http://www.freewebs.com/cesarmerino/Store\%200f\%20pub/TPVNM_rev.pdf.

Masacre, S. y Cantwell, B. (2012). Transatlantic moves to the market: the United States and the European Union. Higher Education, 63 (5), 583-606.

Mateo, J. (2007). Interpretando la realidad, construyendo nuevas formas de conocimiento: el desarrollo competencial y su evaluación. Revista de Investigación Educativa, 25 (2), 513-531. 
McDonald, B. y Boud, D. (2003). The Impact of self-assessment on achievement: the effects of self-assessment training on performance in external examinations. Assessment in Education, 10 (2), 209-220.

Mcgaghie, W. (1991). Professional Competence Evaluation. Educational Researcher, 20 (1), 3-9.

Medina Rivilla, A., Domínguez Garrido, M.C. y Sánchez Romero, C. (2013). Evaluación de las competencias de los estudiantes: modelos y técnicas para la valoración. Revista de Investigación Educativa 31 (1), 239-255.

Menter, I. y Hulme, M. (2012). Teacher education in Scotland - riding out the recession? Educational Research, 54 (2), 149-160.

Moran, A. (2012).Crises as catalysts for change: re-energising teacher education in Northern Ireland. Educational Research, 54 (2), 137-147.

Payne, E. y Brown, G. (2011). Communication and practice with examination criteria. Does this influence performance in examinations? Assessment \& Evaluation In Higher Education, 36 (6), 619-626.

Perrenoud, P. (2004). Diez nuevas competencias para enseñar. Barcelona: Grao.

Pimienta, J.H. (2011). Secuencias didácticas: aprendizaje y evaluación de competencias en educación superior. Bordón, 63 (1), 77-92.

Reddy, Y.M. y Andrade, H. (2010). A review of rubric use in higher education. Assessment \& Evaluation in Higher Education, 35 (4), 435-448.

Rochelle, E., Tractenberg, R.E., Kevin, T. y FitzGerald, K.T. (2012). A Mastery Rubric for the design and evaluation of an institutional curriculum in the responsible conduct of research. Assessment \& Evaluation In Higher Education, 37 (8), 1003-1021.

Rodríguez Espinar, S. (2003). Nuevos retos y enfoques en la formación del profesorado universitario. Revista de Educación, 331, 67-99.

Rodríguez Izquierdo, R.M. (2003). Reaprender a enseñar. Una experiencia de formación para la mejora continua de la docencia universitaria. Revista Interuniversitaria de Formación del Profesorado, 7 (2), 79-84.

Rodríguez, G., Ibarra, M.S. y Gómez, M.A. (2011). e-Autoevaluación en la universidad: un reto para profesores y estudiantes, Revista de Educación, 356, 401-430.

Rubistar. Consultado el 20 de febrero de 2012 en http://rubistar.4teachers.org/index.php.

Rust, C. (2007) Towards a scholarship of assessment, Assessment and Evaluation in Higher Education, 32 (2) $229-237$.

Rust, C., Price, M. y O'Donovan, B. (2003). Improving students' learning by developing their understanding of assessment criteria and processes. Assessment \& Evaluation in Higher Education, 28 (2), 147-164.

Sadler, D. (2009). Indeterminacy in the Use of Preset Criteria for Assessment and Grading. Assessment \& Evaluation in Higher Education 34 (2), 159-79.

Taras, M. (2002). Using assessment for learning and learning from assessment. Assessment and Evaluation in Higher Education, 27 (6), 501-510.

Taras, M. (2010). Student self-assessment: processes and consequences. Teaching in Higher Education, 15 (2), 199-209. 
Tejada, J. (2011). La evaluación de las competencias en contextos no formales: dispositivos e instrumentos de evaluación. Revista de Educación, 354, 731-745.

Timmerman, B.E.C., Strickland, D.C., Johnson, R.L. y Payne, J.R. (2011). Development of a 'universal' rubric for assessing undergraduates' scientific reasoning skills using scientific writing. Assessment \& Evaluation In Higher Education, 36 (5), 509-547.

Torrance, H. (2007). Assessment as Learning? How the use of explicit learning objectives, assessment criteria and feedback in post-secondary education and training can come to dominate learning. Assessment in Education: Principles, Policy \& Practice, 14 (3), 281-94.

Tractenberg, R.E., Umans, J.G. y McCarter, R.J. (2010). A Mastery Rubric: guiding curriculum design, admissions and development of course objectives. Assessment \& Evaluation In Higher Education, 35 (1), 15-32.

Trevitt, C., Breman, E. y Stocks, C. (2012). Assessment and learning: Is it time to rethink student activities and academic roles? Revista de Investigación Educativa, 30 (2), 253269.

Turley, E. y Gallagher, C. (2008). On the Uses of Rubrics: Reframing the Great Rubric Debate. English Journal, 97 (4), 87-92.

Van der Schaaf, M., Baartman, L. y Prins, F. (2012). Exploring the role of assessment criteria during teachers' collaborative judgement processes of students' portfolios. Assessment \& Evaluation in Higher Education, 37 (7), 847-860.

Vandenberg, A., Stollak, M., McKeag, L. y Obermann, D. (2010). GPS in the classroom: using rubrics to increase student achievement. Research in Higher Education Journal, 9, 110. Consultado el 22 de enero de 2013 en http://www.aabri.com/manuscripts/ 10522.pdf.

Villa, A. y Poblete, M. (2011). Evaluación de competencias genéricas: principios, oportunidades y limitaciones. Bordón, 63 (1), 147-170.

Wilson, V.A., y Onwuegbuzie, A.J. (1999). Improving Achievement and Student Satisfaction through Criteria-Based Evaluation: Checklists and Rubrics in Educational Research Courses. Paper presented at Annual Meeting of the Mid-South Educational Research Association. Point Clear, AL.

Zabala, A. y Arnau, L. (2007). 11 ideas clave. Cómo aprender y enseñar competencias. Barcelona: Graó.

Zabalza, M.A. (2003). Competencias docentes del profesorado universitario. Calidad y desarrollo profesional. Madrid: Narcea.

Zabalza, M.A. (2006). Buscando una nueva hoja de ruta en la formación del profesorado. Revista de Educación, 340, 51-58. 


\section{Autores}

Ma Paz García Sanz

Es profesora Titular de Universidad de la Universidad de Murcia. Entre sus líneas de investigación prioritarias, en la actualidad destacan fundamentalmente las siguientes: Planificación, aprendizaje y evaluación de competencias, Evaluación del profesorado, Evaluación de programas educativos y sociales, Evaluación de instituciones educativas y Relaciones familia-centro educativo. Dentro de estas líneas ha dirigido Trabajos Fin de Grado, Trabajos Fin de Máster y Tesis Doctorales; ha colaborado en la realización de variedad de Proyectos de Investigación y de Innovación Educativa, así como en contratos de investigación; ha realizado diferentes ponencias en cursos y talleres de formación del profesorado en distintas Universidades; ha presentado aportaciones en Congresos y Jornadas; ha colaborado en la organización de más de una docena de eventos científicos; y ha realizado variedad de publicaciones en Editoriales y Revistas científicas de reconocido prestigio 